\title{
CONGENITAL KINKING OF THE AORTIC ARCH
}

\author{
BY \\ J. N. PATTINSON AND R. G. GRAINGER \\ From the Radiodiagnostic Departments of the Middlesex, St. Thomas' and the London Chest Hospitals \\ Received February 9, 1959
}

In the past few years, a congenital anomaly of the aortic arch-a kinked or buckled arch-has been described and established as an entity. Although it usually presents a characteristic radiological picture, it is not as yet widely recognized either by radiologists or clinicians. In order to elucidate the plain X-ray findings, the patient is frequently subjected to complicated, unnecessary, and even uncomfortable procedures such as angiography or occasionally exploratory thoracotomy.

To further the more widespread appreciation of the diagnostic radiological appearances, five new cases of congenitally kinked aortic arch are reported and illustrated. Also recorded are the confirmatory results of the specialized techniques that were employed before the typical findings on conventional radiography were recognized.

\section{The ANatomical Deformity}

The normal aortic arch resembles the handle of an umbrella, presenting an even convex loop, inclined at about $30^{\circ}$ to the sagittal plane. In this anomaly, the descending portion of the aortic arch is acutely kinked with an angular convexity directed forwards, downwards, and slightly to the right at the level of the ligamentum arteriosum. The kink occurs at the aortic isthmus, where there is normally a faint indentation on the concavity of the aortic arch immediately proximal to the slight dilatation (aortic spindle) of the upper descending aorta. The aorta proximal to the kink is elongated and rises higher in the mediastinum than usual. The ascending aorta is not dilated and is normally situated. The apex of the abnormal kink is closely applied to the upper and posterior aspect of the left pulmonary artery and left main bronchus, which may be displaced forwards, downwards, and to the right. The œsophagus is also usually displaced forwards and to the right, since it is closely applied to the kinked segment. The aorta immediately distal to the kink may be dilated. It turns sharply backwards and slightly to the left curving downwards into a normally placed descending aorta.

\section{The Radiological Appearance}

Postero-Anterior (P.A.) View. It is in this projection that the anomaly is first detected. The appearances are usually diagnostic, and many of the cases present an identical appearance. There is an oval opacity; less opaque than a normal aortic arch, situated high up on the left superior mediastinal border. It lies higher than the usual aortic arch and therefore is often mistaken for a tumour. This opacity is produced by the abnormally high aortic arch. Overlapping its lower pole is a second opacity of greater radio-density with a sharply defined convex upper border, which continues below into a normally placed descending aorta. This second opacity, although at a rather low level, is frequently misinterpreted as a normal aortic arch. It is in fact an end-on view of the descending aorta immediately distal to the kink. These characteristic features are best seen on a well penetrated P.A. film (Fig. 1 and 3).

Left Anterior Oblique (L.A.O.) View. In this projection the aortic arch is placed parallel to the film. The high aortic arch, the sharp kink forwards at the site of the ligamentum arteriosum, and the backward curve into the normal descending aorta are well demonstrated (Fig. 7). The acute forward angulation of the posterior border of the aorta at the site of the kink is usually well seen, and is a highly suggestive feature. 


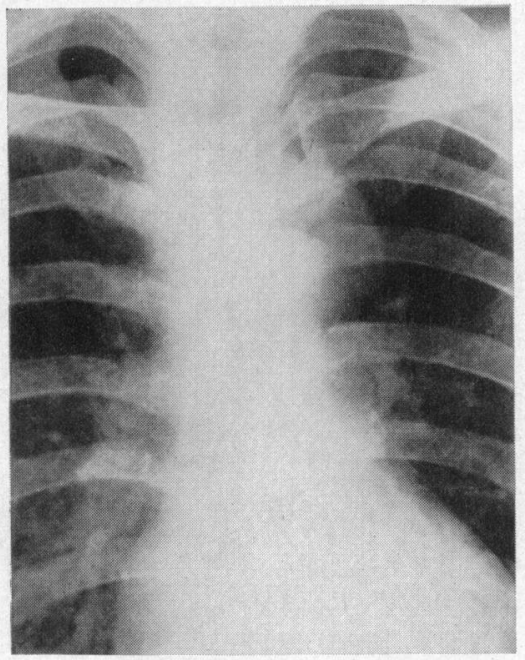

Fig. 1.-Case 1. Man, 40 years. Large opacity of low radio-density above apparent aortic knuckle. The upper opacity is due to the aortic arch; the lower opacity to the "post-kink" segment of the aorta. B.P. normal.

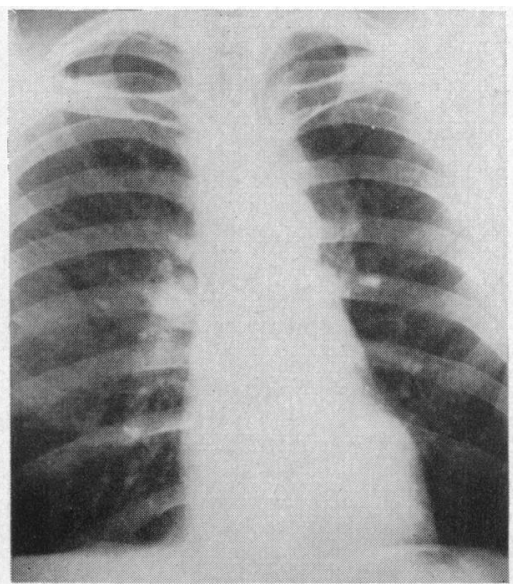

Fig. 3.-Case 2. Man, 38 years. B.P. 135/100. Appearances in P.A. film similar to Fig. 1.

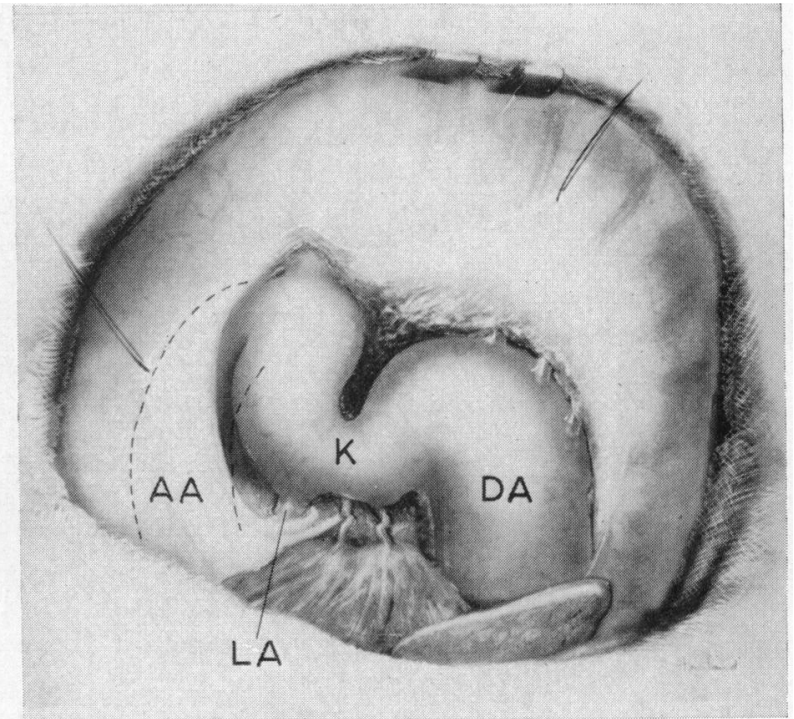

FIG. 2.-Case 1. The appearances at thoracotomy as seen from the left. $\mathrm{AA}=$ Ascending Aorta. $\mathrm{K}=\mathrm{K}$ ink. $\mathrm{LA}=$ Ligamentum Arteriosum. $\mathrm{DA}=$ Descending Aorta.

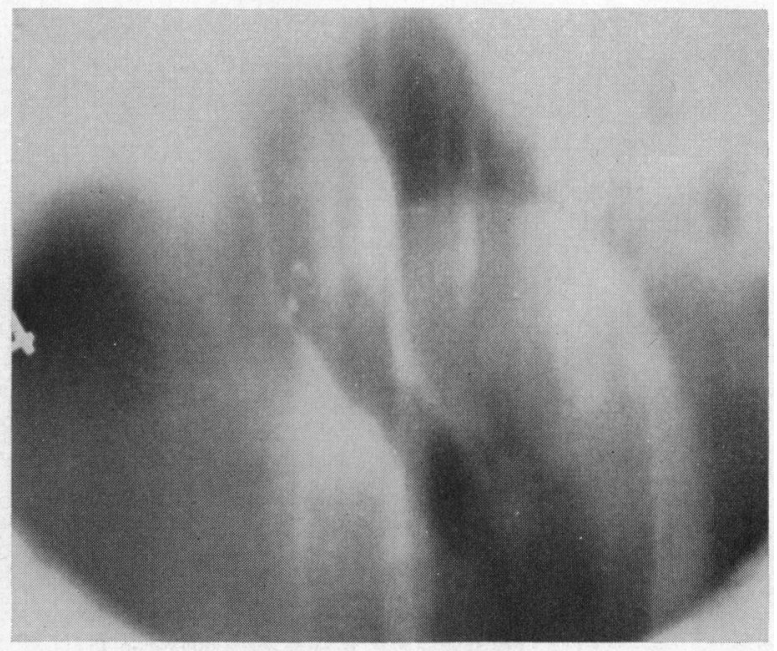

FIG. 4.-Case 2. Lateral tomogram, showing the posterior border of the kink in the aortic arc.

These appearances are often more clearly shown by tomography in this projection (L.A.O.), although such an investigation should rarely be necessary (Fig. 4).

Right Anterior Oblique (R.A.O.) View. The ascending aorta and aortic arch are seen in profile, superimposed on one another as in the normal. The aorta immediately distal to the kink, running horizontally backwards and to the left, produces a dense round shadow behind and below the aortic arch (Fig. 8).

Barium Swallow (P.A.). The unusually high aortic arch causes little or no impression on the esophagus. The aorta immediately distal to the kink deeply indents the left border of the osophagus (Fig. 6) and it is this impression that is frequently mistaken for the normal aortic arch. Occasionally, a long continuous 


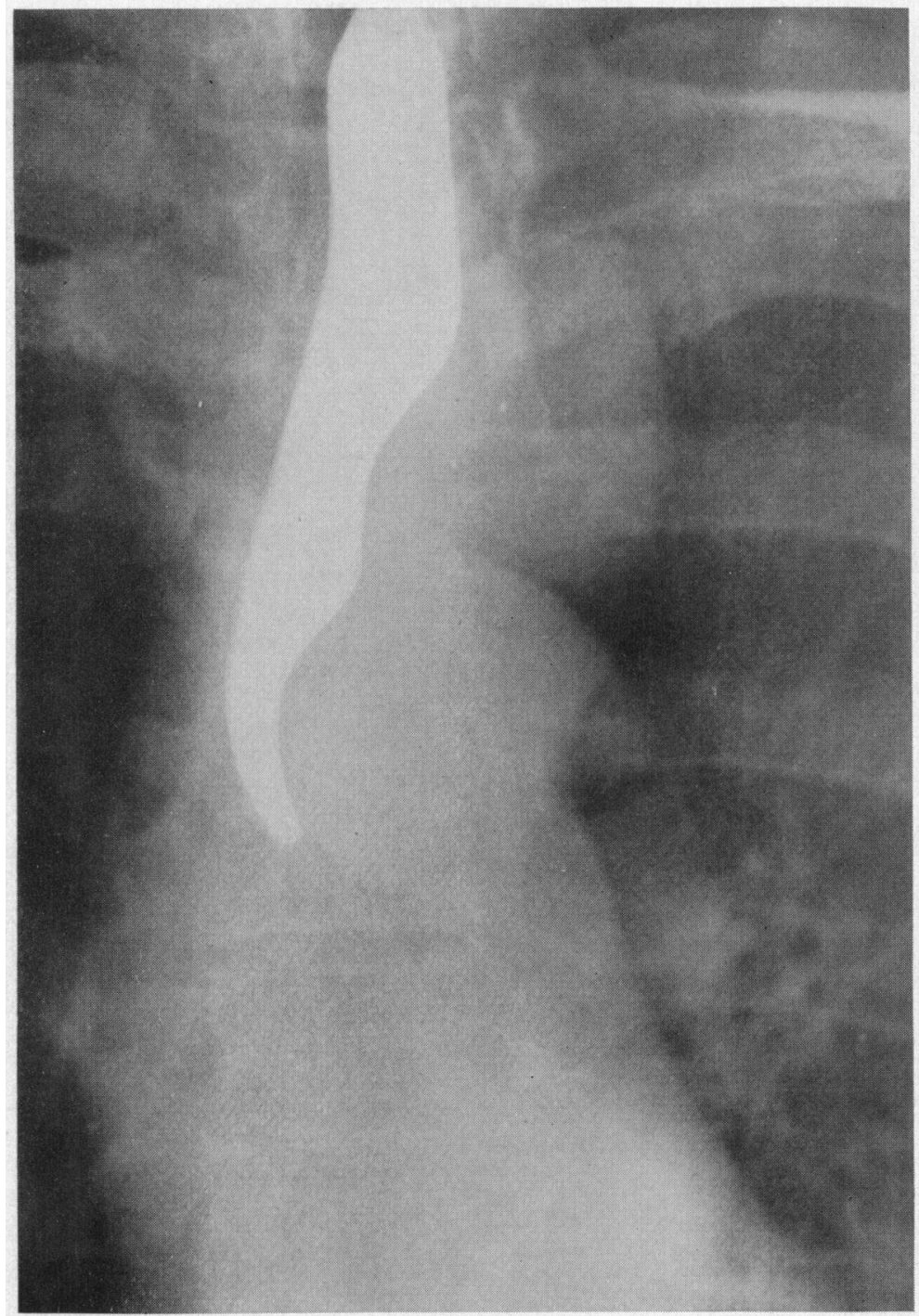

Fig. 5.-Case 3. Barium swallow demonstrating the major cesophageal indentation due to the "post-kink" segment of the aorta, and the minor indentation above it due to the aortic arch.

œsophageal concavity is formed by the combined indentations of the arch and "post-kink" segment of the aorta.

Barium Swallow (L.A.O.). The œsophagus is displaced forwards by the aorta distal to the kink in a similar manner to that produced by the post-stenotic segment in coarctation of the aorta (Fig. 5).

Angiography. The anatomy of the aorta can be well demonstrated by angiocardiography or thoracic aortography (Fig. 6). These examinations should rarely be necessary once the characteristic appearances on conventional radiography have been appreciated.

Simultaneous bi-plane apparatus is preferable, but if this is not available the most valuable single projection is the right posterior oblique (R.P.O.). Slight narrowing of the aortic lumen at the site of the kink may be seen, and slight dilatation of the aorta distal to the kink is not uncommon (Fig. 6). These appearances differ from coarctation in that there is no severe stenosis nor visible collateral circulation. 


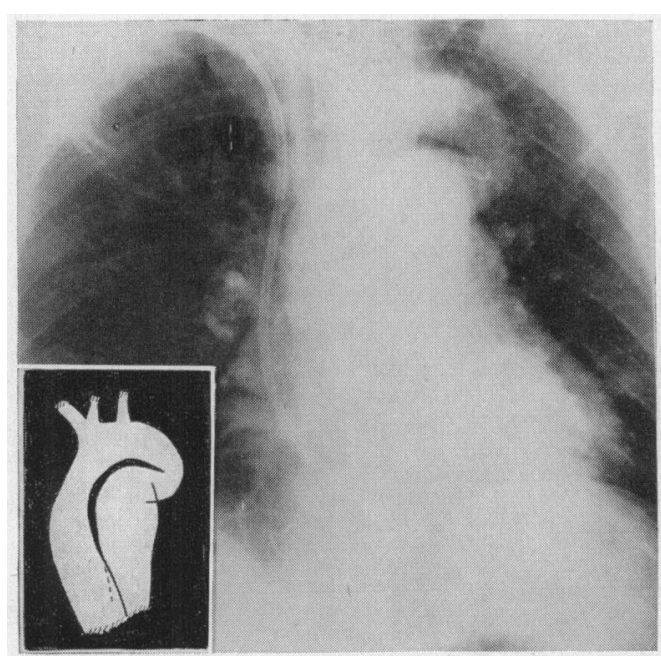

A
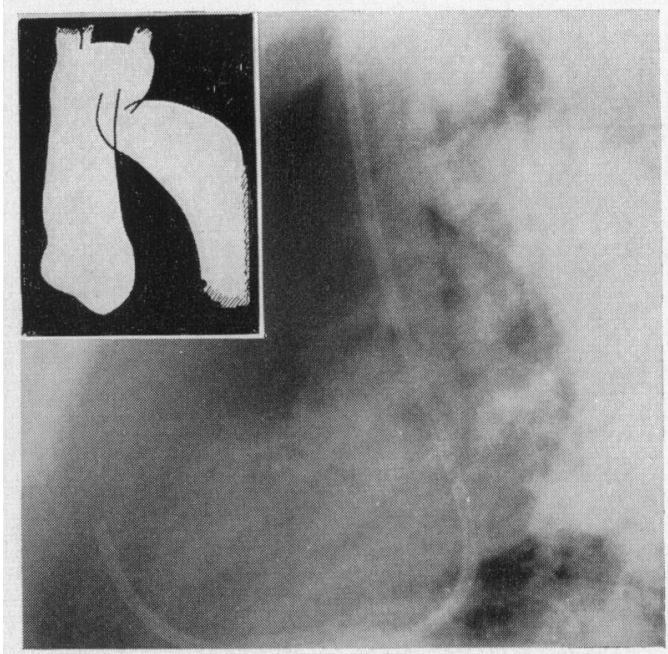

B

Fig. 6.-Case 2. Simultaneous antero-posterior and lateral angiocardiogram, showing the deformity of the aorta. In this patient the aortic arch above the kink is more in the coronal plane than is usual with the "kinked aorta" deformity.

\section{CASE Histories}

Case 1. A man of 40 years, asymptomatic, was found to have an abnormal superior mediastinal shadow (Fig. 1) on a routine P.A. chest film taken elsewhere prior to emigration. He was referred for surgery with a diagnosis of superior mediastinal tumour. A pre-operative film suggested the possibility of coarctation of the aorta, but there were no abnormal physical signs. At thoracotomy, a prominent forward and downward kinking of the distal part of the aortic arch was found. There was no significant narrowing of the aortic lumen, but the aorta distal to the kink was dilated. The ligamentum arteriosum was short and was attached to the apex of the kink (Fig. 2). This obliterated ductus was divided without resultant change in the deformity. Five years later the radiographic appearances remained unchanged.

Case 2. A man, aged 38 years, was found on mass miniature radiography to have an abnormal left superior mediastinum. On direct questioning he admitted to some dyspnœa on exertion and recent dysphagia. On examination, a moderate aortic systolic murmur was heard; brachial and femoral pulses and pressures were normal. A superior mediastinal tumour or aneurysm was suspected, and he was referred from another hospital for angiography. The similarity of the P.A. film (Fig. 3) to that of Case 1 suggested the diagnosis of a kinked aortic arch. This suspicion was strengthened by the appearances on a barium swallow examination and lateral tomography Fig (4). The diagnosis was confirmed by angiocardiography, which showed a high and rather small calibre aortic arch describing a semicircle, almost in the coronal plane (Fig. 6A). There was a $90^{\circ}$ kink at the expected site of the ligamentum arteriosum, distal to which the descending aorta was dilated.

Case 3. A man, aged 51 years, had had a mole on his back since birth. In 1956 it was locally excised because of its recent rapid growth. Three months later it recurred, grew rapidly and was widely excised. Sections revealed a malignant amelanotic melanoma. Metastases appeared in the cervical lymph nodes, and these were removed by block dissection. Enlarged nodes appeared in the axilla and a P.A. film of the chest was interpreted as showing enlarged metastatic superior mediastinal lymph nodes. He was referred to our hospital for radiotherapy. On examination his blood pressure was $135 / 90$ in both arms and the femoral pulses were normal. Conventional radiography, including a barium swallow (Fig. 5), showed the characteristic appearances of a kinked aortic arch. Radiotherapy was therefore confined to the axilla.

Case 4. A man, aged 59 years, who complained of dyspepsia was found to have a gastric ulcer on barium meal examination. During fluoroscopy, an abnormal superior mediastinum was observed. Chest radiographs showed the typical findings of a buckled aortic arch. In addition there was some unfolding of the ascending and descending aorta, probably arteriosclerotic in origin (Fig. 7 and 8).

Case 5. A man, aged 36 years, during an investigation of long standing neurosis, was found to have hypertension, which was labile (varying between $230 / 130$ and 110/70), and thought to be emotional in origin. There was a harsh aortic systolic murmur. Chest radiographs revealed a kinked aortic arch. Kymography showed synchronous pulsation of the aorta both above and below the kink. In this case there is unusual displacement of the kink to the right, with prominent lateral bowing of the descending aorta.

\section{Discussion}

Thirty-seven cases of kinked aortic arch without coarctation have been previously reported or referred to: but some of them may have been reported twice. The abnormality is more frequent than this figure suggests; we have knowledge of, or have seen, several other patients not included in this series. 


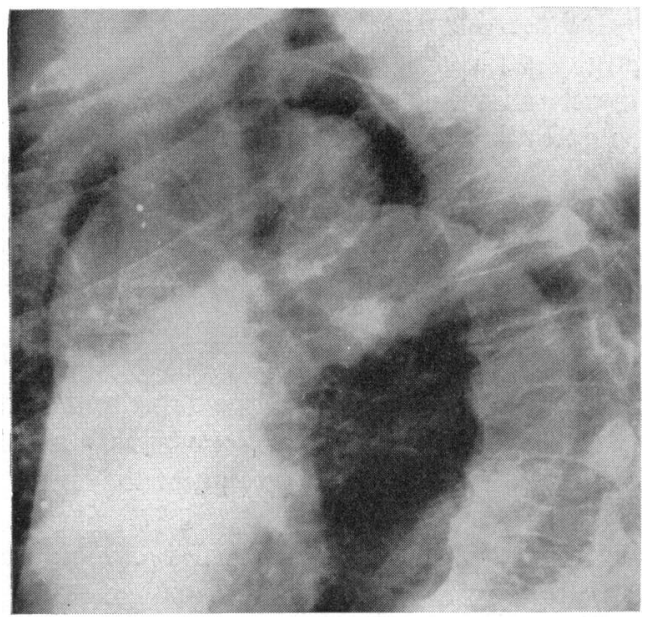

FIG. 7.-Case 4. Male, 59 years. Left anterior oblique film, showing prominent kink plus arteriosclerotic unfolding of the descending aorta.

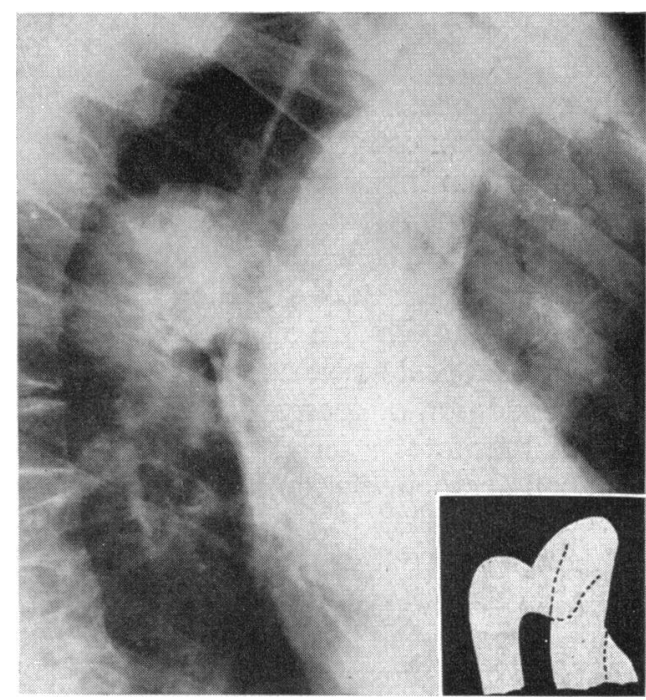

FIG. 8.-Case 4. Right anterior oblique film, showing opacity due to "post-kink" segment behind the aortic arch.

Souders et al. (1951) were the first to appreciate the entity of congenital kinking of the aortic arch without coarctation. They gave the first detailed description of the radiological appearances of this anomaly when reporting three cases. Their first, a 58-year-old man, is particularly instructive as the abnormal radiological appearances were thought to be due to the enlarged superior mediastinal glands of Hodgkin's disease. Radiotherapy had no effect, so thoracotomy was undertaken. A sharply kinked aortic arch was found. The apex of the kink was sited at the insertion of a short, taut, patent ductus arteriosus. There was no evidence of narrowing of the aorta. Division of the ductus (which was thought to be the cause of the kink) did not alter the appearances. Radiography three months later showed no change from the pre-operative films. Their next two cases were investigated by angiography, the third presented atypical features and was not convincingly confirmed.

Reich had previously described, in 1949, the angiographic appearances of this anomaly in a healthy man of 40 years. He believed this to be an example of slight coarctation of the aorta. Dotter and Steinberg (1951) reported two cases of buckled aortic arch without clinical or radiological evidence of coarctation. In 1956 Steinberg, in presenting another example of this anomaly (which was associated with slight dilatation of the aortic sinuses of Valsalva), refers briefly to his experience with 10 cases of buckled arch.

Di Guglielmo and Guttadauro added two further cases demonstrated by angiography in 1955. The second patient, aged 12 years, also had a ventricular septal defect. In the case reported by Vaughan in 1956, the clinical problem was identical with that in our Case 3. His patient had a seminoma of the testis, treated by orchidectomy, but later it metastasized to the para-aortic abdominal glands. A postero-anterior chest film taken to exclude metastases revealed an abnormal opacity over the apparent aortic knuckle. A left anterior oblique film confirmed that this appearance was due to a kinked aortic arch and not due to enlarged lymph glands. Bruwer and Burchell (1956) and Bruwer (1957) reported four cases and stated that a total of eleven had been seen at the Mayo Clinic. Samuel and Morris, in describing two further cases in 1957, advocated tomography in the left anterior oblique position as a simple method of demonstrating the kink in the aortic arch. Stevens (1958) recently reported four more cases under the title of pseudocoarctation, one of these was operated upon for a supposed superior mediastinal tumour. 
The radiological diagnosis of kinked aortic arch is of considerable clinical and practical importance. The most frequent presentation is the finding of an abnormal left superior mediastinal opacity on the postero-anterior chest film. The radiologist must then confirm that this appearance is due to a benign congenital anomaly of the aortic arch and not to a mediastinal tumour.

The mistaken interpretation of the radio-opacity above the apparent aortic knob as a superior mediastinal tumour is a real and dangerous hazard. As described above, several patients have been subjected to therapeutic irradiation and to exploratory thoracotomy, including one of our series (Case 1). An understanding of the anatomy of this congenital anomaly and the recognition of the typical radiological appearances will usually obviate the necessity for these procedures.

The radiological appearances of the kinked aortic arch may be reproduced exactly in the more frequent condition of coarctation of the arch. The absence of hypertension in the arms and head, the normal femoral arterial pressure and wave form, the absence of clinically or radiologically evident collateral circulation, and the normal-sized ascending aorta, will exclude significant coarctation.

Although this differentiation of a kinked arch from a coarcted arch is a valid clinical and radiological one, the two conditions appear to have much in common. It has been suggested that the kinked aorta is an incomplete form of coarctation, in which the obliteration of the aortic lumen is either entirely absent or minimal. The forward displacement of the aorta at the attachment of the ligamentum arteriosum is very similar in the two conditions, but is usually more acute and obvious in the arch that is kinked but not coarcted. The elongation of the aorta proximal to the kink is more prominent, and the post-kink or post-stenotic dilatation is absent or less striking in these patients without significant stenosis.

To justify the diagnosis of "kinked aortic arch" there must be no clinical evidence of aortic narrowing. Several patients have in fact been shown on angiography or thoracotomy to have minimal asymptomatic narrowing of the aortic lumen at the kink, with slight distal dilatation. There is therefore something to be said on anatomical grounds for using the terms "sub-clinical coarctation," "atypical coarctation," or even "pseudo-coarctation." But, as the usual presentation of the clinical problem is the differential diagnosis of a left superior mediastinal opacity, these names are not really satisfactory. The term "kinked" or "buckled" aortic arch is an accurate anatomical description and implies the benign nature of the anomaly.

The presence of a loud basal heart murmur may suggest congenital heart disease, as emphasized by Bruwer and Burchell (1956). This is presumably due to the turbulence of the blood flow through the sharply kinked aorta. The occasional occurrence of co-existing congenital heart lesions must be borne in mind, as patent ductus arteriosus, ventricular septal defect, and dilatation of the aortic sinuses have been described in association with a kinked aortic arch.

Aneurysms of the aortic arch (particularly the congenital variety associated with a patent ductus) may occasionally produce rather similar appearances in the postero-anterior chest film, but show other characteristic features (Rigler, 1955).

The "kinked aortic arch" is believed to be a congenital malformation associated with a short, taut, ligamentum arteriosum, and an unduly long aortic arch segment. We do not know which of these two factors is the fundamental anomaly.

Barry (1951) has described the important changes that occur in the embryonic aortic arch complex during development. Some arterial segments elongate while others become greatly compressed. One of the most striking changes is the cephalic shift in the origin of the subclavian arteries. These develop from the 7 th dorsal intersegmental arteries. With the descent of the heart into the thorax, their origin moves far cephalad and is accompanied by prominent compression of the 3rd to 7th segments of the dorsal aortic root and of the 4th aortic arch segments. Failure of this normal compression of the left 4th aortic arch and 3rd to 7th dorsal aortic root segments during development, would result in abnormal length of the arch of the aorta, which therefore kinks at the fixed point, namely the insertion of the ligamentum arteriosum. Many of the published angiograms of the kinked aortic arch show that the distance between the origins of the left common 
carotid and left subclavian arteries is greater than normal. Poker et al. (1958) have also commented on this fact. This increased distance between the origin of the two arteries would result from the failure of normal compression of certain embryonic aortic segments as suggested above.

In the development of "kinked carotid artery," Parkinson et al. (1939) suggested that while there may be a congenital element, the more important causative factors are hypertension, arteriosclerosis, kyphosis, and an elongated unfolded aortic arch. The kinking of the congenitally buckled aortic arch will be increased by the same elongation of the aorta which may occur in later life from hypertension and arteriosclerosis (Case 4). The accentuation of the radiological appearances produced by these secondary changes may account for the fact that in the majority of recorded cases of kinked aortic arch, the age of the patient has been 30 years or more.

The deformity bears a close similarity to coarctation of the aorta situated at the ligamentum arteriosum, the essential difference being the occurrence of clinically significant stenosis in the latter.

\section{SUMMARY}

Five new cases of kinked aortic arch without coarctation are described and illustrated. The correct diagnosis can usually be made by conventional radiography. The typical appearances are described in detail. The radiological diagnosis is of practical importance in order to avoid the mistaken interpretation of mediastinal tumour. The congenital defect responsible for the anatomical anomaly is discussed.

We are grateful to our clinical colleagues for their kind permission to report the clinical data relating to these patients.

\section{REFERENCES}

Barry, A. (1951). Anat. Rec., 221, 111.

Bruwer, A. J., and Burchell, H. B. (1956). J. Amer. med. Ass., 162, 1445. (1957). Brit. J. Radiol., 30, 387.

Di Guglielmo, L., and Guttadauro, M. (1955). Acta radiol., Stockh., 44, 121.

Dotter, C. T., and Steinberg, I. (1951). Angiocardiography. Ann. Rantgenol., $20,181$.

Parkinson, J., Bedford, D. E., and Almond, S. (1939). Brit. Heart J., 1, 345.

Poker, N., Finby, N., and Steinberg, I. (1958). Amer. J. Roentgenol., 80, 193.

Reich, N. E. (1949). Diseases of the Aorta. The Macmillan Co., New York. P. 60.

Rigler, R. G. (1955). Quoted by Bruwer, A. J., and Burchell, H. B. (1956).

Samuel, E., and Morris, L. (1957). Medicine in South Africa. P. 74.

Steinberg, I. (1956). Brit. Heart J., 18, 85.

Stevens, G. M. (1958). Radiology, 70, 67.

Souders, C. R., Pearson, C. M., and Adams, H. D. (1951). Dis. Chest., 20, 35.

Vaughan, B. F. (1956). Brit. J. Radiol., 30, 387. 